hep-th/0212039

December 2002

Revised version: February 2003

\title{
Nonlinear Self-Duality and Supergravity
}

\author{
Sergei M. Kuzenko and Shane A. McCarthy \\ School of Physics, The University of Western Australia, \\ 35 Stirling Highway, Crawley W.A. 6009, Australia \\ kuzenko@cyllene.uwa.edu.au, shane@physics.uwa.edu.au
}

\begin{abstract}
The concept of self-dual supersymmetric nonlinear electrodynamics is generalized to a curved superspace of $\mathcal{N}=1$ supergravity, for both the old minimal and the new minimal versions of $\mathcal{N}=1$ supergravity. We derive the self-duality equation, which has to be satisfied by the action functional of any $\mathrm{U}(1)$ duality invariant model of a massless vector multiplet, and construct a family of self-dual nonlinear models. This family includes a curved superspace extension of the $\mathcal{N}=1$ super Born-Infeld action. The supercurrent and supertrace in such models are proved to be duality invariant. The most interesting and unexpected result is that the requirement of nonlinear self-duality yields nontrivial couplings of the vector multiplet to Kähler sigma models. We explicitly derive the couplings to general Kähler sigma models in the case when the matter chiral multiplets are inert under the duality rotations, and more specifically to the dilaton-axion chiral multiplet when the group of duality rotations is enhanced to $\mathrm{SL}(2, \mathbb{R})$.
\end{abstract}




\section{Introduction}

In 1935, Schrödinger [1] showed that the nonlinear electrodynamics of Born and Infeld [2], proposed as a new fundamental theory of the electromagnetic field, possessed a remarkable property - invariance under $\mathrm{U}(1)$ duality rotations. Although the great expectations, which originally led the authors of [2] to put forward their model, never came true, the Born-Infeld action has re-appeared in the spotlight since the 1980's as a low energy effective action in string theory $[3,4]$. Along with patterns of duality in extended supergravity $[5,6]$, this motivated Gaillard and Zumino, Gibbons and others [7, 8, 9, 10, 11, 12, 13] to develop a general theory of (nonlinear) self-duality in four and higher space-time dimensions for non-supersymmetric theories ${ }^{1}$. Extension to $4 \mathrm{D} \mathcal{N}=1,2$ globally supersymmetric theories was given in $[15,16]$. As a final step in developing the formalism, it was also shown in [17] how to re-formulate the requirement of nonlinear self-duality (i.e. a nonlinear equation which the action functional has to satisfy in order for the theory to be duality invariant) as a condition of manifest invariance of the interaction; in a sense, this is a nice extension of Schrödinger's ideas [1].

There exist deep yet mysterious connections between nonlinear self-duality and supersymmetry and here we give three examples. First, in the case of partial spontaneous supersymmetry breakdown $\mathcal{N}=2 \rightarrow \mathcal{N}=1$, the Maxwell-Goldstone multiplet $[18,20]$ (coinciding with the $\mathcal{N}=1$ supersymmetric Born-Infeld action [21]) and the tensor Goldstone multiplet $[19,20]$ were shown in $[15,16]$ to be self-dual, i.e. invariant under $\mathrm{U}(1)$ duality rotations. Our second example concerns partial supersymmetry breakdown $\mathcal{N}=4 \rightarrow \mathcal{N}=2$. To construct a Maxwell-Goldstone multiplet action for such a scenario - the $\mathcal{N}=2$ supersymmetric Born-Infeld action ${ }^{2}$ - it was suggested in [16] to look for an $\mathcal{N}=2$ vector multiplet action which should be (i) self-dual; (ii) invariant under a nonlinearly realized central charge bosonic symmetry. These requirements turn out to allow one to restore the Goldstone multiplet action uniquely to any fixed order in powers of chiral superfield strength $\mathcal{W}$; this was carried out in [16] up to order $\mathcal{W}^{10}$. Recently, there has been considerable progress in developing the formalism of nonlinear realizations to describe the partial SUSY breaking $\mathcal{N}=4 \rightarrow \mathcal{N}=2$ [23]. So far, the authors of [23] have reproduced the action obtained in [16]. Finally, we should mention that the (Coulomb branch) low energy effective action of the $\mathcal{N}=4$ supersymmetric Yang-Mills theory is conjectured to be invariant under $\mathrm{U}(1)$ duality rotations [15] (a weaker form of

\footnotetext{
${ }^{1}$ Properties of nonlinear electrodynamics in curved space from the viewpoint of dualities were studied in $[14]$.

${ }^{2}$ See $[22]$ for earlier attempts to construct an $\mathcal{N}=2$ supersymmetric version of the Born-Infeld action.
} 
self-duality of the effective action was proposed in [24]).

The above features provide enough evidence for considering supersymmetric self-dual systems to be quite interesting and their properties worth studying. In the present note, self-dual nonlinear supersymmetric electrodynamics $[15,16]$ is coupled to $\mathcal{N}=1$ supergravity in the presence of nonlinear Kähler sigma models. To describe $\mathcal{N}=1$ supergravity, we separately consider its two off-shell realizations ${ }^{3}$ : the old minimal formulation $[25,26]$ and the new minimal formulation [27]. By now, $4 \mathrm{D} \mathcal{N}=1$ superfield supergravity is a subject of several textbooks $[29,30,31]$ to which the interested reader is referred for further details and references.

The structure of the paper is as follows. Working with the old minimal version of $\mathcal{N}=1$ supergravity, in section 2 we derive the self-duality equation as the condition for a vector multiplet model to be invariant under $\mathrm{U}(1)$ duality rotations. We then demonstrate self-duality under a superfield Legendre transformation, introduce a family of self-dual nonlinear models and argue that the supercurrent and supertrace in such models are duality invariant. To a large extent, these results are just a minimal curved superspace extension of the globally supersymmetric results presented in [16]. However, the game will become more interesting when we turn to the couplings to new minimal supergravity and Kähler sigma models. Section 3 is devoted to the coupling of selfdual nonlinear supersymmetric electrodynamics to the dilaton-axion multiplet in curved superspace; this is of interest from the point of view of string theory.

\section{Electromagnetic duality rotations in curved super- space}

We follow the notation ${ }^{4}$ and $\mathcal{N}=1$ supergravity conventions of [31]. Unless otherwise stated we work with the old minimal formulation of $\mathcal{N}=1$ supergravity. The superspace geometry is described by covariant derivatives

$$
\begin{aligned}
& \mathcal{D}_{A}=\left(\mathcal{D}_{a}, \mathcal{D}_{\alpha}, \overline{\mathcal{D}}^{\dot{\alpha}}\right)=E_{A}+\Omega_{A}, \\
& E_{A}=E_{A}{ }^{M} \partial_{M}, \quad \Omega_{A}=\frac{1}{2} \Omega_{A}{ }^{b c} M_{b c}=\Omega_{A}{ }^{\beta \gamma} M_{\beta \gamma}+\Omega_{A} \dot{\beta} \dot{\gamma} \bar{M}_{\dot{\beta} \dot{\gamma}},
\end{aligned}
$$

\footnotetext{
${ }^{3}$ The non-minimal version of $\mathcal{N}=1$ supergravity [28] does not lead to interesting matter couplings, see e.g. [31].

${ }^{4}$ In particular, $z^{M}=\left(x^{m}, \theta^{\mu}, \bar{\theta}_{\dot{\mu}}\right)$ are the coordinates of $\mathcal{N}=1$ curved superspace, $\mathrm{d}^{8} z=\mathrm{d}^{4} x \mathrm{~d}^{2} \theta \mathrm{d}^{2} \bar{\theta}$ is the full flat superspace measure, and $\mathrm{d}^{6} z=\mathrm{d}^{4} x \mathrm{~d}^{2} \theta$ is the measure in the chiral subspace.
} 
with $E_{A}{ }^{M}$ the supervielbein, $\Omega_{A}$ the Lorentz superconnection and $M_{b c} \Leftrightarrow\left(M_{\beta \gamma}, \bar{M}_{\dot{\beta} \dot{\gamma}}\right)$ the Lorentz generators. The covariant derivatives obey the following algebra:

$$
\begin{gathered}
\left\{\mathcal{D}_{\alpha}, \overline{\mathcal{D}}_{\dot{\alpha}}\right\}=-2 \mathrm{i} \mathcal{D}_{\alpha \dot{\alpha}}, \\
\left\{\mathcal{D}_{\alpha}, \mathcal{D}_{\beta}\right\}=-4 \bar{R} M_{\alpha \beta}, \quad\left\{\overline{\mathcal{D}}_{\dot{\alpha}}, \overline{\mathcal{D}}_{\dot{\beta}}\right\}=-4 R \bar{M}_{\dot{\alpha} \dot{\beta}}, \\
{\left[\overline{\mathcal{D}}_{\dot{\alpha}}, \mathcal{D}_{\beta \dot{\beta}}\right]=-\mathrm{i} \varepsilon_{\dot{\alpha} \dot{\beta}}\left(R \mathcal{D}_{\beta}+G_{\beta}{ }^{\dot{\gamma}} \overline{\mathcal{D}}_{\dot{\gamma}}-\left(\overline{\mathcal{D}}^{\dot{\gamma}} G_{\beta}{ }^{\dot{\delta}}\right) \bar{M}_{\dot{\gamma} \dot{\delta}}+2 W_{\beta}{ }^{\gamma \delta} M_{\gamma \delta}\right)-\mathrm{i}\left(\mathcal{D}_{\beta} R\right) \bar{M}_{\dot{\alpha} \dot{\beta}},} \\
{\left[\mathcal{D}_{\alpha}, \mathcal{D}_{\beta \dot{\beta}}\right]=\mathrm{i} \varepsilon_{\alpha \beta}\left(\bar{R} \overline{\mathcal{D}}_{\dot{\beta}}+G_{\dot{\beta}}^{\gamma} \mathcal{D}_{\gamma}-\left(\mathcal{D}^{\gamma} G_{\dot{\beta}}^{\delta}\right) M_{\gamma \delta}+2 \bar{W}_{\dot{\beta}}^{\dot{\gamma} \dot{\delta}} \bar{M}_{\dot{\gamma} \dot{\delta}}\right)+\mathrm{i}\left(\overline{\mathcal{D}}_{\dot{\beta}} \bar{R}\right) M_{\alpha \beta},}
\end{gathered}
$$

where the tensors $R, G_{a}=\bar{G}_{a}$ and $W_{\alpha \beta \gamma}=W_{(\alpha \beta \gamma)}$ satisfy the Bianchi identities

$$
\overline{\mathcal{D}}_{\dot{\alpha}} R=\overline{\mathcal{D}}_{\dot{\alpha}} W_{\alpha \beta \gamma}=0, \quad \overline{\mathcal{D}}^{\dot{\gamma}} G_{\alpha \dot{\gamma}}=\mathcal{D}_{\alpha} R, \quad \mathcal{D}^{\gamma} W_{\alpha \beta \gamma}=\mathrm{i} \mathcal{D}_{(\alpha}{ }^{\dot{\gamma}} G_{\beta) \dot{\gamma}} .
$$

Modulo purely gauge degrees of freedom, all geometric objects - the supervielbein and the superconnection - can be expressed in terms of three unconstrained superfields (known as the prepotentials of old minimal supergravity): gravitational superfield $H^{m}=\bar{H}^{m}$, chiral compensator $\varphi\left(\bar{E}_{\dot{\alpha}} \varphi=0\right)$ and its conjugate $\bar{\varphi}$. The old minimal supergravity action is

$$
S_{\mathrm{SG}, \text { old }}=-3 \int \mathrm{d}^{8} z E^{-1}, \quad E=\operatorname{Ber}\left(E_{A}^{M}\right),
$$

with the gravitational coupling constant being set equal to one.

In what follows, to simplify notation, we introduce

$$
A \cdot B=\int \mathrm{d}^{8} z \frac{E^{-1}}{R} A^{\alpha} B_{\alpha}, \quad \bar{A} \cdot \bar{B}=\int \mathrm{d}^{8} z \frac{E^{-1}}{\bar{R}} \bar{A}_{\dot{\alpha}} \bar{B}^{\dot{\alpha}},
$$

with $A_{\alpha}$ and $B_{\alpha}$ covariantly chiral spinor superfields, $\overline{\mathcal{D}}_{\dot{\alpha}} A_{\alpha}=\overline{\mathcal{D}}_{\dot{\alpha}} B_{\alpha}=0$ (a similar notation will also be used for chiral scalars). We often make use of the relation

$$
\begin{aligned}
\int \mathrm{d}^{8} z E^{-1} \mathcal{L} & =-\frac{1}{4} \int \mathrm{d}^{8} z \frac{E^{-1}}{R}\left(\overline{\mathcal{D}}^{2}-4 R\right) \mathcal{L} \\
& =-\frac{1}{4} \int \mathrm{d}^{6} z \varphi^{3}\left(\overline{\mathcal{D}}^{2}-4 R\right) \mathcal{L},
\end{aligned}
$$

where the equality in the last line takes place in the so-called chiral representation (see $[29,31]$ for more details). This result is especially simple in the chiral case, $\mathcal{L}=\mathcal{L}_{\mathrm{c}} / R$, with $\mathcal{L}_{\mathrm{c}}$ a covariantly chiral scalar, $\overline{\mathcal{D}}_{\dot{\alpha}} \mathcal{L}_{\mathrm{c}}=0$.

\section{$2.1 \quad$ Self-duality equation}

Consider a model of a single Abelian $\mathcal{N}=1$ vector multiplet in curved superspace as generated by the action $S[W, \bar{W}]$. The covariantly (anti) chiral spinor superfield strengths 
$\bar{W}_{\dot{\alpha}}$ and $W_{\alpha}$,

$$
W_{\alpha}=-\frac{1}{4}\left(\overline{\mathcal{D}}^{2}-4 R\right) \mathcal{D}_{\alpha} V, \quad \bar{W}_{\dot{\alpha}}=-\frac{1}{4}\left(\mathcal{D}^{2}-4 \bar{R}\right) \overline{\mathcal{D}}_{\dot{\alpha}} V,
$$

are defined in terms of a real unconstrained prepotential $V$. Consequently, the strengths are constrained superfields satisfying the Bianchi identity

$$
\mathcal{D}^{\alpha} W_{\alpha}=\overline{\mathcal{D}}_{\dot{\alpha}} \bar{W}^{\dot{\alpha}} .
$$

Suppose that $S[W, \bar{W}] \equiv S[v]$ can be unambiguously defined ${ }^{5}$ as a functional of unconstrained (anti) chiral superfields $\bar{W}_{\dot{\alpha}}$ and $W_{\alpha}$. Then, one can define covariantly (anti) chiral superfields $\bar{M}_{\dot{\alpha}}$ and $M_{\alpha}$ as

$$
\text { i } M_{\alpha}[v] \equiv 2 \frac{\delta}{\delta W^{\alpha}} S[v], \quad-\text { i } \bar{M}^{\dot{\alpha}}[v] \equiv 2 \frac{\delta}{\delta \bar{W}_{\dot{\alpha}}} S[v],
$$

with the functional derivatives defined by

$$
\delta S=\delta W \cdot \frac{\delta S}{\delta W}+\delta \bar{W} \cdot \frac{\delta S}{\delta \bar{W}} .
$$

The vector multiplet equation of motion following from the action $S[W, \bar{W}]$ reads

$$
\mathcal{D}^{\alpha} M_{\alpha}=\overline{\mathcal{D}}_{\dot{\alpha}} \bar{M}^{\dot{\alpha}} \text {. }
$$

Since the Bianchi identity (2.8) and the equation of motion (2.11) have the same functional form, one may consider $\mathrm{U}(1)$ duality rotations

$$
\left(\begin{array}{c}
M_{\alpha}^{\prime}\left[v^{\prime}\right] \\
W_{\alpha}^{\prime}
\end{array}\right)=\left(\begin{array}{rr}
\cos \tau & -\sin \tau \\
\sin \tau & \cos \tau
\end{array}\right)\left(\begin{array}{c}
M_{\alpha}[v] \\
W_{\alpha}
\end{array}\right),
$$

where $M^{\prime}$ should be

$$
\text { i } M_{\alpha}^{\prime}\left[v^{\prime}\right]=2 \frac{\delta}{\delta W^{\prime \alpha}} S\left[v^{\prime}\right] .
$$

Following the method described in $[15,16]$, the condition that $S[W, \bar{W}]$ be self-dual is equivalent to the reality condition

$$
\operatorname{Im}(W \cdot W+M \cdot M)=0 .
$$

Any solution, $S[W, \bar{W}]$, of this self-duality equation generates a $\mathrm{U}(1)$ duality invariant supersymmetric electrodynamics coupled to old minimal supergravity. The self-duality equation can be shown to be equivalent to the following invariance condition under $\mathrm{U}(1)$ duality rotations $(2.12)$

$$
S\left[v^{\prime}\right]-\frac{\mathrm{i}}{4}\left(W^{\prime} \cdot M^{\prime}\left[v^{\prime}\right]-\bar{W}^{\prime} \cdot \bar{M}^{\prime}\left[v^{\prime}\right]\right)=S[v]-\frac{\mathrm{i}}{4}(W \cdot M[v]-\bar{W} \cdot \bar{M}[v]) .
$$

\footnotetext{
${ }^{5}$ As indicated in $[15,16]$, this is always possible if $S[W, \bar{W}]$ does not involve the combination $\mathcal{D}^{\alpha} W_{\alpha}$ as an independent variable.
} 


\subsection{Invariance under superfield Legendre transformation}

One of the nice properties of all models of self-dual electrodynamics is invariance under Legendre transformation [12]. It was shown in [15] that this property also holds for any globally supersymmetric model of the massless vector multiplet that is invariant under $\mathrm{U}(1)$ duality rotations. We will demonstrate that this property also naturally extends to curved superspace.

Consider a massless vector multiplet model in curved superspace described by the action $S[W, \bar{W}]$. The Legendre transformation is defined by introducing an auxiliary action

$$
S\left[\mathcal{W}, \overline{\mathcal{W}}, W_{\mathrm{D}}, \bar{W}_{\mathrm{D}}\right]=S[\mathcal{W}, \overline{\mathcal{W}}]-\frac{\mathrm{i}}{2}\left(\mathcal{W} \cdot W_{\mathrm{D}}-\overline{\mathcal{W}} \cdot \bar{W}_{\mathrm{D}}\right)
$$

where $\mathcal{W}_{\alpha}$ is now an unconstrained covariantly chiral spinor superfield, and $W_{\mathrm{D} \alpha}$ the dual field strength

$$
W_{\mathrm{D} \alpha}=-\frac{1}{4}\left(\overline{\mathcal{D}}^{2}-4 R\right) \mathcal{D}_{\alpha} V_{\mathrm{D}}, \quad \bar{W}_{\mathrm{D} \dot{\alpha}}=-\frac{1}{4}\left(\mathcal{D}^{2}-4 \bar{R}\right) \overline{\mathcal{D}}_{\dot{\alpha}} V_{\mathrm{D}},
$$

with the Lagrange multiplier $V_{\mathrm{D}}$ a real scalar superfield. This model is equivalent to the original model, since upon elimination of $W_{\mathrm{D}}$ by its equation of motion we regain $S[\mathcal{W}, \overline{\mathcal{W}}]$, with the condition that $\mathcal{W}$ satisfy the Bianchi identity (2.8). However, we may instead eliminate $\mathcal{W}$ by its equation of motion, in which case we obtain a dual action $S_{\mathrm{D}}\left[W_{\mathrm{D}}, \bar{W}_{\mathrm{D}}\right]$, the Legendre transform of $S[W, \bar{W}]$. Now, let $S[W, \bar{W}]$ be a solution of the self-duality equation (2.14), and hence it satisfies the invariance condition (2.15) under arbitrary $\mathrm{U}(1)$ duality rotations (2.12). Then, eq. (2.15) for a finite duality rotation $\tau=\pi / 2$ is easily seen to be equivalent to the statement that $S_{\mathrm{D}}=S$.

\subsection{Family of self-dual models}

Extending the globally supersymmetric results of [15], we now present a family of $\mathcal{N}=1$ supersymmetric self-dual models with actions of the general form

$$
S[W, \bar{W}]=\frac{1}{4} \int \mathrm{d}^{8} z \frac{E^{-1}}{R} W^{2}+\frac{1}{4} \int \mathrm{d}^{8} z \frac{E^{-1}}{\bar{R}} \bar{W}^{2}+\frac{1}{4} \int \mathrm{d}^{8} z E^{-1} W^{2} \bar{W}^{2} \Lambda(u, \bar{u}),
$$

where $\Lambda(u, \bar{u})$ is a real analytic function of the complex variable

$$
u \equiv \frac{1}{8}\left(\mathcal{D}^{2}-4 \bar{R}\right) W^{2} .
$$


The condition of self-duality (2.14) on the model (2.18) turns out to be equivalent to a differential equation which the interaction $\Lambda(u, \bar{u})$ has to satisfy. This equation is

$$
\operatorname{Im}\left\{\Gamma-\bar{u} \Gamma^{2}\right\}=0, \quad \Gamma=\frac{\partial(u \Lambda)}{\partial u} .
$$

As an important example, consider a minimal curved superspace extension ${ }^{6}$ of the $\mathcal{N}=1$ supersymmetric Born-Infeld action $[21,18,20]$

$$
S_{\mathrm{BI}}=\frac{1}{4} \int \mathrm{d}^{8} z \frac{E^{-1}}{R} X+\frac{1}{4} \int \mathrm{d}^{8} z \frac{E^{-1}}{\bar{R}} \bar{X}
$$

where the covariantly chiral scalar $X$ is a functional of $W_{\alpha}$ and $\bar{W}_{\dot{\alpha}}$ defined by the nonlinear constraint

$$
X+\frac{1}{4} X\left(\overline{\mathcal{D}}^{2}-4 R\right) \bar{X}=W^{2}
$$

Following [18], this can be shown to be equivalent to

$$
S_{\mathrm{BI}}=\frac{1}{4} \int \mathrm{d}^{8} z \frac{E^{-1}}{R} W^{2}+\frac{1}{4} \int \mathrm{d}^{8} z \frac{E^{-1}}{\bar{R}} \bar{W}^{2}+\int \mathrm{d}^{8} z E^{-1} \frac{W^{2} \bar{W}^{2}}{1+\frac{1}{2} A+\sqrt{1+A+\frac{1}{4} B^{2}}},
$$

where

$$
A=4(u+\bar{u}), \quad B=4(u-\bar{u}) .
$$

This action is of the form (2.18), and one can readily check that the differential equation (2.20) is satisfied. Therefore, the minimal curved superspace extension of the $\mathcal{N}=1$ super Born-Infeld action is self-dual.

\subsection{Duality invariance of the supercurrent and supertrace}

In the bosonic case, self-dual models have the important property that the energymomentum tensor is invariant under $\mathrm{U}(1)$ duality rotations $[1,7,9,10,12]$. It is natural to ask whether this property extends to the supersymmetric case, the superfield generalization of the energy-momentum tensor being the supercurrent $T_{a}=\bar{T}_{a}$ and supertrace $T$, $\overline{\mathcal{D}}_{\dot{\alpha}} T=0$. These are defined in terms of covariantized variational derivatives with respect to the supergravity prepotentials (see $[29,31]$ for more details),

$$
T_{a}=\frac{\Delta S}{\Delta H^{a}}, \quad T=\frac{\Delta S}{\Delta \varphi},
$$

\footnotetext{
${ }^{6}$ Such a curved superspace action was discussed in [32].
} 
and satisfy the conservation equation

$$
\overline{\mathcal{D}}^{\dot{\alpha}} T_{\alpha \dot{\alpha}}=-\frac{2}{3} \mathcal{D}_{\alpha} T
$$

when the matter superfields are put on the mass shell.

Gaillard and Zumino [7, 12] developed an elegant, model-independent proof of the fact that the energy-momentum tensor of any self-dual bosonic system is invariant under $\mathrm{U}(1)$ duality rotations. It is not quite trivial however to generalize this proof to the supersymmetric case, and this is why we will follow a brute-force approach, similar to $[1,9,10]$, and directly check duality invariance of the supercurrent and supertrace.

Let us first turn to the supertrace. For the model (2.18), it is

$$
T=\frac{1}{8} W^{2}\left(\overline{\mathcal{D}}^{2}-4 R\right)\left[\bar{W}^{2}(\Gamma+\bar{\Gamma}-\Lambda)\right],
$$

with $\Gamma$ defined in (2.20). Consider an infinitesimal duality rotation $\delta W_{\alpha}=\tau M_{\alpha}, \delta M_{\alpha}=$ $-\tau W_{\alpha}$, where

$$
\text { i } M_{\alpha}=W_{\alpha}\left\{1-\frac{1}{4}\left(\overline{\mathcal{D}}^{2}-4 R\right)\left[\bar{W}^{2}\left(\Lambda+\frac{1}{8}\left(\mathcal{D}^{2}-4 \bar{R}\right)\left(W^{2} \frac{\partial \Lambda}{\partial u}\right)\right)\right]\right\} \text {. }
$$

It is an instructive exercise to show that $\delta T$ vanishes for $\Lambda \neq 0$ only if the self-duality equation (2.20) is taken into account. Now, the conservation equation (2.26) is to be satisfied both before and after applying the duality rotation. Since $T$ is duality invariant, the left hand side of (2.26) should also be invariant. This essentially implies duality invariance of the supercurrent.

Turning now to the supercurrent, with the use of the techniques described in [31], we find

$$
\begin{aligned}
T_{\alpha \dot{\alpha}} & =\mathrm{i} M_{\alpha} \bar{W}_{\dot{\alpha}}-\mathrm{i} W_{\alpha} \bar{M}_{\dot{\alpha}}-\frac{\mathrm{i}}{4} \mathcal{D}_{\alpha \dot{\alpha}}\left(W^{2} \bar{W}^{2}(\Gamma-\bar{\Gamma})\right) \\
& -\frac{1}{6} G_{\alpha \dot{\alpha}} W^{2} \bar{W}^{2}(\Gamma+\bar{\Gamma}-\Lambda)-\frac{1}{24}\left[\mathcal{D}_{\alpha}, \overline{\mathcal{D}}_{\dot{\alpha}}\right]\left(W^{2} \bar{W}^{2}(\Gamma+\bar{\Gamma}-\Lambda)\right) \\
& -\frac{\mathrm{i}}{4}\left(W^{2} \overleftrightarrow{\mathcal{D}_{\alpha \dot{\alpha}}} \bar{W}^{2}\right) \Lambda-\frac{\mathrm{i}}{4} W^{2} \bar{W}^{2}\left(\mathcal{D}_{\alpha \dot{\alpha}} u\right) \frac{\partial \Lambda}{\partial u}+\frac{\mathrm{i}}{4} W^{2} \bar{W}^{2}\left(\mathcal{D}_{\alpha \dot{\alpha}} \bar{u}\right) \frac{\partial \Lambda}{\partial \bar{u}} \\
& +\frac{\mathrm{i}}{16}\left(\mathcal{D}_{\alpha \dot{\alpha}} W^{2}\right) \bar{W}^{2}\left(\mathcal{D}^{2}-4 \bar{R}\right)\left(W^{2} \frac{\partial \Lambda}{\partial u}\right)-\frac{\mathrm{i}}{16} W^{2}\left(\mathcal{D}_{\alpha \dot{\alpha}} \bar{W}^{2}\right)\left(\overline{\mathcal{D}}^{2}-4 R\right)\left(\bar{W}^{2} \frac{\partial \Lambda}{\partial \bar{u}}\right)
\end{aligned}
$$

Off the mass shell, the variational derivative $\Delta S / \Delta H$ can be shown to include the extra (gauge non-invariant) term

$$
\frac{\mathrm{i}}{4}\left(\mathcal{D}^{\beta} M_{\beta}-\overline{\mathcal{D}}_{\dot{\beta}} \bar{M}^{\dot{\beta}}\right)\left[\mathcal{D}_{\alpha}, \overline{\mathcal{D}}_{\dot{\alpha}}\right] V
$$


which involves the naked prepotential $V$ and therefore does not allow a naive generalization of the Gaillard-Zumino proof $[7,12]$ to superspace.

A tedious calculation is required to explicitly show that (i) the conservation equation (2.26) is indeed satisfied; and (ii) the supercurrent (2.29) is duality invariant. When performing these calculations, the following equivalent expression for the supercurrent is often easier to work with:

$$
\begin{aligned}
T_{\alpha \dot{\alpha}} & =\mathrm{i} M_{\alpha} \bar{W}_{\dot{\alpha}}-\mathrm{i} W_{\alpha} \bar{M}_{\dot{\alpha}}+\frac{\mathrm{i}}{4} \mathcal{D}_{\alpha \dot{\alpha}}\left(W^{2} \bar{W}^{2}(\Gamma+\bar{\Gamma}-\Lambda)\right) \\
& -\frac{1}{6} G_{\alpha \dot{\alpha}} W^{2} \bar{W}^{2}(\Gamma+\bar{\Gamma}-\Lambda)-\frac{1}{24}\left[\mathcal{D}_{\alpha}, \overline{\mathcal{D}}_{\dot{\alpha}}\right]\left(W^{2} \bar{W}^{2}(\Gamma+\bar{\Gamma}-\Lambda)\right) \\
& -\frac{\mathrm{i}}{2} W^{2} \mathcal{D}_{\alpha \dot{\alpha}}\left[\bar{W}^{2}\left(\Lambda+\frac{1}{8}\left(\mathcal{D}^{2}-4 \bar{R}\right)\left(W^{2} \frac{\partial \Lambda}{\partial u}\right)\right)\right] \\
& +\frac{\mathrm{i}}{2} W^{2} \bar{W}^{2}\left(\mathcal{D}_{\alpha \dot{\alpha}} \bar{u}\right) \frac{\partial \Lambda}{\partial \bar{u}}-\frac{\mathrm{i}}{16} W^{2}\left(\mathcal{D}_{\alpha \dot{\alpha}} \bar{W}^{2}\right)\left(\overline{\mathcal{D}}^{2}-4 R\right)\left(\bar{W}^{2} \frac{\partial \Lambda}{\partial \bar{u}}\right) .
\end{aligned}
$$

\subsection{Coupling to new minimal supergravity}

It is known that new minimal supergravity can be treated as a super-Weyl invariant dynamical system describing the coupling of old minimal supergravity to a real covariantly linear scalar superfield $\mathbb{L}$,

$$
\left(\overline{\mathcal{D}}^{2}-4 R\right) \mathbb{L}=\left(\mathcal{D}^{2}-4 \bar{R}\right) \mathbb{L}=0
$$

Any system of matter superfields $\Psi$ coupled to new minimal supergravity can be treated as a super-Weyl invariant coupling of old minimal supergravity to the matter superfields $\Psi$ and $\mathbb{L}$ (see [31] for a review). It is clear that matter couplings in the new minimal formulation of supergravity are more restrictive as compared to the old minimal version. Here we will demonstrate how to couple the models of self-dual nonlinear electrodynamics to new minimal supergravity.

Super-Weyl transformations, originally introduced in [33], are simply local rescalings of the chiral compensator in old minimal supergravity (see [29, 31]). In terms of the covariant derivatives, the super-Weyl transformation ${ }^{7}$ is

$$
\mathcal{D}_{\alpha} \rightarrow \mathrm{e}^{\sigma / 2-\bar{\sigma}}\left(\mathcal{D}_{\alpha}-\left(\mathcal{D}^{\beta} \sigma\right) M_{\alpha \beta}\right), \quad \overline{\mathcal{D}}_{\dot{\alpha}} \rightarrow \mathrm{e}^{\bar{\sigma} / 2-\sigma}\left(\overline{\mathcal{D}}_{\dot{\alpha}}-\left(\overline{\mathcal{D}}^{\dot{\beta}} \bar{\sigma}\right) \bar{M}_{\dot{\beta} \dot{\alpha}}\right)
$$

\footnotetext{
${ }^{7}$ Under (2.33), the full superspace measure changes as $\mathrm{d}^{8} z E^{-1} \rightarrow \mathrm{d}^{8} z E^{-1} \exp (\sigma+\bar{\sigma})$, while the chiral superspace measure transforms as $\mathrm{d}^{8} z E^{-1} / R \rightarrow \mathrm{d}^{8} z\left(E^{-1} / R\right) \exp (3 \sigma)$, see eq. (2.6).
} 
with $\sigma(z)$ an arbitrary covariantly chiral scalar parameter, $\overline{\mathcal{D}}_{\dot{\alpha}} \sigma=0$. Since

$$
\left(\mathcal{D}^{2}-4 \bar{R}\right) \rightarrow \mathrm{e}^{-2 \bar{\sigma}}\left(\mathcal{D}^{2}-4 \bar{R}\right) \mathrm{e}^{\sigma}
$$

when acting on a scalar superfield, it is clear that the super-Weyl transformation law of $\mathbb{L}$ is uniquely fixed to be

$$
\mathbb{L} \rightarrow \mathrm{e}^{-\sigma-\bar{\sigma}} \mathbb{L}
$$

The new minimal supergravity action ${ }^{8}$ is

$$
S_{\mathrm{SG}, \text { new }}=3 \int \mathrm{d}^{8} z E^{-1} \mathbb{L} \ln \mathbb{L} .
$$

Given a massless vector multiplet, eq. (2.7), the gauge field $V$ is inert under the super-Weyl transformations, while $W_{\alpha}$ changes as

$$
W_{\alpha} \rightarrow \mathrm{e}^{-3 \sigma / 2} W_{\alpha}
$$

It is then clear that only the kinetic term in (2.18) is super-Weyl invariant. However, the nonlinear part of (2.18) can be promoted to a super-Weyl invariant functional by coupling the vector multiplet to $\mathbb{L}$. To achieve this, it is sufficient to notice that the combination ${ }^{9}$

$$
\left(\mathcal{D}^{2}-4 \bar{R}\right)\left(\frac{W^{2}}{\mathbb{L}^{2}}\right)
$$

is super-Weyl invariant. As a result, we can replace the action (2.18) by the following functional $^{10}$

$$
\begin{aligned}
S[W, \bar{W}, \mathbb{L}]=\frac{1}{4} \int \mathrm{d}^{8} z \frac{E^{-1}}{R} W^{2} & +\frac{1}{4} \int \mathrm{d}^{8} z \frac{E^{-1}}{\bar{R}} \bar{W}^{2} \\
& +\frac{1}{4} \int \mathrm{d}^{8} z E^{-1} \frac{W^{2} \bar{W}^{2}}{\mathbb{L}^{2}} \Lambda\left(\frac{u}{\mathbb{L}^{2}}, \frac{\bar{u}}{\mathbb{L}^{2}}\right),
\end{aligned}
$$

which is (i) super-Weyl invariant and (ii) self-dual, i.e. it solves the self-duality equation (2.14). This action describes self-dual supersymmetric electrodynamics in new minimal supergravity.

\footnotetext{
${ }^{8}$ In the flat superspace limit, when we set $H^{m}=0$ and $\varphi=1$, such an action describes the so-called improved tensor multiplet [34].

${ }^{9}$ Here we actually generalize the construction [15] of $\mathcal{N}=1$ superconformal U(1) duality invariant systems in flat superspace.

${ }^{10}$ Without spoiling the super-Weyl invariance and self-duality of the action (2.39), the 'compensator' $\mathbb{L}$ can be replaced in (2.39) by $\mathbb{L} / \kappa$, with $\kappa$ a coupling constant. We set this constant to be one since it can be absorbed via renormalization of the self-interaction, $\hat{\Lambda}(u, \bar{u})=\kappa^{2} \Lambda\left(\kappa^{2} u, \kappa^{2} \bar{u}\right)$, see [16].
} 


\subsection{Coupling to nonlinear sigma models}

As is known, supersymmetric nonlinear sigma models are most easily described in the framework of new minimal supergravity (see, e.g. [31] for a review). Given a Kähler manifold parametrized by complex coordinates $\phi$ and their conjugates $\bar{\phi}$, with $K(\phi, \bar{\phi})$ the Kähler potential, the corresponding supergravity-matter action is

$$
S=3 \int \mathrm{d}^{8} z E^{-1} \mathbb{L} \ln \mathbb{L}+\int \mathrm{d}^{8} z E^{-1} \mathbb{L} K(\phi, \bar{\phi}) .
$$

The dynamical variables $\phi$ are covariantly chiral scalar superfields, $\overline{\mathcal{D}}_{\dot{\alpha}} \phi=0$, being inert with respect to the super-Weyl transformations. The action is obviously super-Weyl invariant. Moreover, the action is invariant under the Kähler transformations

$$
K(\phi, \bar{\phi}) \rightarrow K(\phi, \bar{\phi})+\lambda(\phi)+\bar{\lambda}(\bar{\phi}),
$$

with $\lambda(\phi)$ an arbitrary holomorphic function.

It is easy to couple the above model to the self-dual supersymmetric electrodynamics (2.39). The supergravity-matter system is described by the action

$$
S[W, \bar{W}, \phi, \bar{\phi}, \mathbb{L}]=3 \int \mathrm{d}^{8} z E^{-1} \mathbb{L} \ln \mathbb{L}+\int \mathrm{d}^{8} z E^{-1} \mathbb{L} K(\phi, \bar{\phi})+S[W, \bar{W}, \mathbb{L}],
$$

and this theory possesses several important symmetries: (i) super-Weyl invariance; (ii) Kähler invariance; (iii) U(1) duality invariance. We should now uncover the description of this theory in the framework of old minimal supergravity.

Let us replace the action (2.42) by the following auxiliary action

$$
S[W, \bar{W}, \phi, \bar{\phi}, \mathbb{L}, U]=3 \int \mathrm{d}^{8} z E^{-1}(U \mathbb{L}-\Upsilon)+S[W, \bar{W}, \Upsilon],
$$

where

$$
\Upsilon=\exp \left(U-\frac{1}{3} K(\phi, \bar{\phi})\right) .
$$

Here the additional dynamical variable $U$ is an unconstrained real scalar superfield, and the action $S[W, \bar{W}, \Upsilon]$ is obtained from (2.39) by replacing $\mathbb{L} \rightarrow \Upsilon$. In order for the action to be super-Weyl invariant, the superfield $U$ must possess the following superWeyl transformation law:

$$
U \rightarrow U-\sigma-\bar{\sigma}
$$

The theories (2.42) and (2.43) are equivalent to each other. Indeed, the $U$-equation of motion derived from (2.43) is algebraic and it can be uniquely solved by expressing 
$U$ in terms of the other superfields. Upon elimination of $U$ in this way we regain the action (2.42). On the other hand, let us consider the $\mathbb{L}$-equation of motion derived from (2.43): $\left(\overline{\mathcal{D}}^{2}-4 R\right) \mathcal{D}_{\alpha} U=0$. The general solution to this equation is just the requirement that $U$ be the sum of a covariantly chiral scalar superfield and its conjugate; as a result, the linear superfield $\mathbb{L}$ completely decouples. Now, the super-Weyl gauge freedom (2.45) allows us to impose the gauge condition $U=0$, and the action (2.43) then becomes

$$
S[W, \bar{W}, \phi, \bar{\phi}]=-3 \int \mathrm{d}^{8} z E^{-1} \mathrm{e}^{-\frac{1}{3} K(\phi, \bar{\phi})}+S\left[W, \bar{W}, \mathrm{e}^{-\frac{1}{3} K(\phi, \bar{\phi})}\right] .
$$

This is the old minimal supergravity counterpart of the model (2.42). To preserve the super-Weyl gauge condition $U=0$, any Kähler transformation (2.41) must now be accompanied by the induced super-Weyl transformation with $\sigma=\frac{1}{3} \lambda(\phi)$. As a result, one ends up with the so-called super-Weyl-Kähler transformations (see [29, 30, 31] for more details).

\section{Coupling to the dilaton-axion multiplet}

In the above analysis of the coupling of self-dual supersymmetric electrodynamics to Kähler sigma models in curved superspace, it was assumed that the matter superfields, $\phi$ and $\bar{\phi}$, are inert under the electromagnetic duality rotations. Of some interest is a more general situation when, say, a chiral matter superfield $\Phi$ and its conjugate $\bar{\Phi}$ do transform under duality rotations, which can now span a larger group than the one corresponding to the pure gauge field case. Coupling to the so-called dilaton-axion supermultiplet is an important example.

We start by formulating the conditions of duality invariance for the Abelian vector multiplet $\left(W_{\alpha}, \bar{W}_{\dot{\alpha}}\right)$ interacting with chiral matter $(\Phi, \bar{\Phi})$ in curved superspace. Let $S[v]=S[W, \bar{W}, \Phi, \bar{\Phi}]$ be the action functional of the supergravity-matter systems, with the dependence of $S[v]$ on the supergravity prepotentials being implicit. We again introduce covariantly (anti) chiral spinor superfields $\bar{M}^{\dot{\alpha}}$ and $M_{\alpha}$ defined by the rule (2.9). Since the Bianchi identity $\mathcal{D}^{\alpha} W_{\alpha}=\overline{\mathcal{D}}_{\dot{\alpha}} \bar{W}^{\dot{\alpha}}$ and the gauge field equation of motion $\mathcal{D}^{\alpha} M_{\alpha}=\overline{\mathcal{D}}_{\dot{\alpha}} \bar{M}^{\dot{\alpha}}$ are of the same functional form, we may consider infinitesimal duality transformations

$$
\delta\left(\begin{array}{l}
M_{\alpha} \\
W_{\alpha}
\end{array}\right)=\left(\begin{array}{ll}
a & b \\
c & d
\end{array}\right)\left(\begin{array}{l}
M_{\alpha} \\
W_{\alpha}
\end{array}\right), \quad \delta \Phi=\xi(\Phi),
$$

with $\xi(\Phi)$ a holomorphic function and $a, b, c$ and $d$ real numbers. 
Following [16], the conditions of duality invariance in the presence of matter can be shown to be

$$
\begin{aligned}
\delta \Phi \cdot \frac{\delta S}{\delta \Phi}+\delta \bar{\Phi} \cdot \frac{\delta S}{\delta \bar{\Phi}} & =\frac{\mathrm{i}}{4} b(W \cdot W-\bar{W} \cdot \bar{W})-\frac{\mathrm{i}}{4} c(M \cdot M-\bar{M} \cdot \bar{M}) \\
& +\frac{\mathrm{i}}{2} a(W \cdot M-\bar{W} \cdot \bar{M}),
\end{aligned}
$$

with $d=-a$. We see that the maximal group of duality transformations is $\operatorname{Sp}(2, \mathbb{R}) \cong$ $\operatorname{SL}(2, \mathbb{R})$. The complex variable $\Phi$ should then parametrize the homogeneous space $\operatorname{SL}(2, \mathbb{R}) / \mathrm{U}(1)$, with the vector field $\xi(\Phi)$ in $(3.2)$ generating the action of $\operatorname{SL}(2, \mathbb{R})$ on the coset space. The matter-free case, which was considered before, corresponds to freezing the superfield $\Phi(z)$ to a given point of the space $\operatorname{SL}(2, \mathbb{R}) / \mathrm{U}(1)$. In such a case, the duality group, $\mathrm{SL}(2, \mathbb{R})$, reduces to $\mathrm{U}(1)$ - the stabilizer of the point chosen.

To describe the dilaton-axion multiplet, we make use of the lower half-plane realization of the coset space $\mathrm{SL}(2, \mathbb{R}) / \mathrm{U}(1)$. Then, the variation $\delta \Phi=\xi(\Phi)$ in (3.1) is

$$
\delta \Phi=b+2 a \Phi-c \Phi^{2}
$$

Our solution to the equations (3.2) reads

$$
\begin{aligned}
S= & 3 \int \mathrm{d}^{8} z E^{-1} \mathbb{L} \ln \mathbb{L}+\int \mathrm{d}^{8} z E^{-1} \mathbb{L}(\mathcal{K}(\Phi, \bar{\Phi})+K(\phi, \bar{\phi})) \\
& +\frac{\mathrm{i}}{4} \int \mathrm{d}^{8} z \frac{E^{-1}}{R} \Phi W^{2}-\frac{\mathrm{i}}{4} \int \mathrm{d}^{8} z \frac{E^{-1}}{\bar{R}} \bar{\Phi} \bar{W}^{2} \\
& -\frac{1}{16} \int \mathrm{d}^{8} z E^{-1}(\Phi-\bar{\Phi})^{2} \frac{W^{2} \bar{W}^{2}}{\mathbb{L}^{2}} \Lambda\left(\frac{\mathrm{i}}{2}(\Phi-\bar{\Phi}) \frac{u}{\mathbb{L}^{2}}, \frac{\mathrm{i}}{2}(\Phi-\bar{\Phi}) \frac{\bar{u}}{\mathbb{L}^{2}}\right),
\end{aligned}
$$

and $u$ is defined in (2.19). Here $\mathcal{K}(\Phi, \bar{\Phi})$ is the Kähler potential of the Kähler manifold $\mathrm{SL}(2, \mathbb{R}) / \mathrm{U}(1)$. The term $\int \mathrm{d}^{8} z E^{-1} \mathbb{L} K(\phi, \bar{\phi})$ in $(3.4)$ corresponds to the chiral matter which is inert under the duality rotations. For $\Phi=-\mathrm{i}$, the action (3.4) reduces to (2.42).

The supergravity-matter system (3.4) enjoys the following important properties: (i) super-Weyl invariance; (ii) Kähler invariance; (iii) $\mathrm{SL}(2, \mathbb{R})$ duality invariance. To reformulate this theory in the framework of the old minimal version of $\mathcal{N}=1$ supergravity, one should eliminate the real linear compensator $\mathbb{L}$ following the procedure described in subsection 2.6. This will lead to

$$
\begin{aligned}
S= & -3 \int \mathrm{d}^{8} z E^{-1} \exp \left(-\frac{1}{3} \mathcal{K}(\Phi, \bar{\Phi})-\frac{1}{3} K(\phi, \bar{\phi})\right) \\
& +\frac{\mathrm{i}}{4} \int \mathrm{d}^{8} z \frac{E^{-1}}{R} \Phi W^{2}-\frac{\mathrm{i}}{4} \int \mathrm{d}^{8} z \frac{E^{-1}}{\bar{R}} \bar{\Phi} \bar{W}^{2} \\
& -\frac{1}{16} \int \mathrm{d}^{8} z E^{-1}(\Phi-\bar{\Phi})^{2} \frac{W^{2} \bar{W}^{2}}{\Upsilon^{2}} \Lambda\left(\frac{\mathrm{i}}{2}(\Phi-\bar{\Phi}) \frac{u}{\Upsilon^{2}}, \frac{\mathrm{i}}{2}(\Phi-\bar{\Phi}) \frac{\bar{u}}{\Upsilon^{2}}\right),
\end{aligned}
$$


where

$$
\Upsilon=\exp \left(-\frac{1}{3} \mathcal{K}(\Phi, \bar{\Phi})-\frac{1}{3} K(\phi, \bar{\phi})\right) .
$$

Unlike (3.4), this action enjoys the super-Weyl-Kähler invariance.

To describe the dilaton-axion complex, we have used the $\mathcal{N}=1$ chiral multiplet. In the context of heterotic string theory, the dilaton-axion complex is realized in terms of the $\mathcal{N}=1$ tensor multiplet. Transition from the chiral to the tensor realization can be implemented as follows. The dilaton-axion Kähler potential can be chosen to be $\mathcal{K}(\Phi, \bar{\Phi})=-\kappa^{2} \ln \mathrm{i}(\Phi-\bar{\Phi})$, with $\kappa$ a constant. As a result, the action (3.4) can be brought (at the cost of sacrificing the manifest gauge invariance in the second line of the action) to such a form that $\Phi$ and $\bar{\Phi}$ appear only in the real combination $\mathrm{i}(\Phi-\bar{\Phi})$. We can then apply a superfield Legendre transformation which turns the description in terms of $\Phi$ and $\bar{\Phi}$ into the one in terms of a real superfield $\mathbb{G}$ under the modified linearity condition

$$
\left(\overline{\mathcal{D}}^{2}-4 R\right) \mathbb{G}=W^{\alpha} W_{\alpha}, \quad\left(\mathcal{D}^{2}-4 \bar{R}\right) \mathbb{G}=\bar{W}_{\dot{\alpha}} \bar{W}^{\dot{\alpha}} .
$$

This constraint is known to describe the Chern-Simons coupling of the tensor multiplet to the vector multiplet. An interesting open question is: What is the fate of the $\operatorname{SL}(2, \mathbb{R})$ duality symmetry in this dual version of the theory?

Acknowledgements. We are grateful to Ian McArthur for discussions and for reading the manuscript. The work of SK is supported by the Australian Research Council, the Australian Academy of Science as well as by UWA research grants. The work of SMc is supported by the Hackett Postgraduate Scholarship.

\section{References}

[1] E. Schrödinger, Proc. Roy. Soc. A150 (1935) 465.

[2] M. Born and L. Infeld, Proc. Roy. Soc. A144 (1934) 425.

[3] E.S. Fradkin and A.A. Tseytlin, Phys. Lett. B163 (1985) 123.

[4] R.G. Leigh, Mod. Phys. Lett. A4 (1989) 2767.

[5] S. Ferrara, J. Scherk and B. Zumino, Nucl. Phys. B121 (1977) 393.

[6] E. Cremmer and B. Julia, Nucl. Phys. B159 (1979) 141. 
[7] M.K. Gaillard and B. Zumino, Nucl. Phys. B193 (1981) 221

[8] B. Zumino, in Quantum Structure of Space and Time, M. J. Duff and C. J. Isham (Eds.), Cambridge University Press, 1982 p. 363.

[9] G.W. Gibbons and D.A. Rasheed, Nucl. Phys. B454 (1995) 185 [hep-th/9506035].

[10] G.W. Gibbons and D.A. Rasheed, Phys. Lett. B365 (1996) 46 [hep-th/9509141].

[11] M.K. Gaillard and B. Zumino, in Supersymmetry and Quantum Field Theory, J. Wess and V.P. Akulov (Eds.), Springer Verlag, 1998, p. 121 [hep-th/9705226].

[12] M.K. Gaillard and B. Zumino, in Duality and Supersymmetric Theories, D.I. Olive and P.C. West (Eds.), Cambridge University Press, 1999, p. 33 [hep-th/9712103].

[13] Y. Tanii, Introduction to supergravities in diverse dimensions, hep-th/9802138; M. Araki and Y. Tanii, Int. J. Mod. Phys. A14 (1999) 1139 [hep-th/9808029]; T. Kimura and I. Oda, Int. J. Mod. Phys. A16 (2001) 503 [hep-th/9904019]; D. Brace, B. Morariu and B. Zumino, in The Many Faces of the Superworld: Yury Golfand Memorial Volume, M. Shifman (Ed.), World Scientific, 2000, p. 103 [hep-th/9905218]; P. Aschieri, D. Brace, B. Morariu and B. Zumino, Nucl. Phys. B574 (2000) 551 [hepth/9909021]; M. Hatsuda, K. Kamimura and S. Sekiya, Nucl. Phys. B561 (1999) 341 [hep-th/9906103]; P. Aschieri, D. Brace, B. Morariu and B. Zumino, Nucl. Phys. B588 (2000) 521 [hep-th/0003228].

[14] G.W. Gibbons and K. Hashimoto, JHEP 0009 (2000) 013 [arXiv:hep-th/0007019].

[15] S.M. Kuzenko and S. Theisen, JHEP 0003 (2000) 034 [hep-th/0001068].

[16] S.M. Kuzenko and S. Theisen, Fortsch. Phys. 49 (2001) 273 [hep-th/0007231].

[17] E.A. Ivanov and B.M. Zupnik, Nucl. Phys. B618 (2001) 3 [hep-th/0110074]; New representation for Lagrangians of self-dual nonlinear electrodynamics, hep-th/0202203.

[18] J. Bagger and A. Galperin, Phys. Rev. D55 (1997) 1091 [hep-th/9608177].

[19] J. Bagger and A. Galperin, Phys. Lett. B412 (1997) 296 [hep-th/9707061].

[20] M. Roček and A.A. Tseytlin, Phys. Rev. D59 (1999) 106001 [hep-th/9811232].

[21] S. Cecotti and S. Ferrara, Phys. Lett. B187 (1987) 335. 
[22] S.V. Ketov, Nucl. Phys. B553 (1999) 250 [hep-th/9812051]; Class. Quant. Grav. 17 (2000) L91 [hep-th/0005126].

[23] S. Bellucci, E. Ivanov and S. Krivonos, Phys. Lett. B502 (2001) 279 [hepth/0012236]; Phys. Rev. D64 (2001) 025014 [hep-th/0101195]; Nucl. Phys. Proc. Suppl. 102 (2001) 26 [hep-th/0103136].

[24] F. Gonzalez-Rey, B. Kulik, I. Y. Park and M. Roček, Nucl. Phys. B544 (1999) 218 [hep-th/9810152].

[25] J. Wess and B. Zumino, Phys. Lett. B66 (1977) 361; R. Grimm, J. Wess and B. Zumino, Phys. Lett. B73 (1978) 415; J. Wess and B. Zumino, Phys. Lett. B74 (1978) 51.

[26] K.S. Stelle and P.C. West, Phys. Lett. B74 (1978) 330; S. Ferrara and P. van Nieuwenhuizen, Phys. Lett. B74 (1978) 333.

[27] V.P. Akulov, D.V. Volkov and V.A. Soroka, Teor. Mat. Fiz. 31 (1977) 12; M.F. Sohnius and P.C. West, Phys. Lett. B105 (1981) 353.

[28] P. Breitenlohner, Nucl. Phys. B124 (1977) 500; W. Siegel and S.J. Gates, Nucl. Phys. B147 (1979) 77.

[29] S.J. Gates, M.T. Grisaru, M. Roček and W. Siegel, Superspace, or One Thousand and One Lessons in Supersymmetry, Front. Phys. 58 (1983) 1 [hep-th/0108200].

[30] J. Wess and J. Bagger, Supersymmetry and Supergravity, Princeton Univ. Press, 1992.

[31] I.L. Buchbinder and S.M. Kuzenko, Ideas and Methods of Supersymmetry and Supergravity or a Walk Through Superspace, IOP, Bristol, 1998.

[32] S.J. Gates and S.V. Ketov, Class. Quant. Grav. 18 (2001) 3561 [hep-th/0104223].

[33] P.S. Howe and R.W. Tucker, Phys. Lett. B80 (1978) 138.

[34] B. de Wit and M. Roček, Phys. Lett. B109 (1982) 439. 